ISSN: 1130-3743 - eISSN: 2386-5660

DOI: http://dx.doi.org/10.14201/teoredu302223246

\title{
PARTICIPACIÓN COMUNITARIA EN EDUCACIÓN- RECONFIGURACIONES DE LO ESCOLAR Y DE LA PARTICIPACIÓN SOCIAL
}

\author{
Community participation in education-reconfigurations \\ of school and social participation
}

\section{Participation communautaire en éducation- reconfigurations de l'école et de la participation sociale}

Yolanda JimÉnEz NARANJO* y Maike KREISEL **

* Universidad Autónoma Benito Juárez de Oaxaca. Instituto de Investigaciones Sociológicas. Avda Universidad, s/n. C.P 68120, Colonia 5 señores. Oaxaca, México.yolanaranjo@gmail.com

** Arbeitsbereich Interkulturelle Bildung. Institut für Bildung im Kindes-und Jugendalter. Fachbereich 5: Erziehungswissenschaften. Universität KoblenzLandau. Campus Landau. Thomas-Nast-Str. 4476829 Landau/Pfalz, Alemania

Fecha de recepción: enero de 2018

Fecha de aceptación: marzo de 2018

RESUMEN

La participación social en educación es un campo vasto en experiencias y perspectivas. En este artículo, pretendemos aportar a dicho debate desde una experiencia educativa específica en escuelas secundarias comunitarias en México, en la cual la participación rebasa formas instrumentales de entender el conocimiento y el apoyo familiar a las escuelas, por un lado, y, por otro, discutimos la relación escuela-comunidad como un eje a partir del cual fomentar otras formas de comprender la educación escolarizada, sus pedagogías y aprendizajes. Sostenemos que formas activas 
de participación social en educación y no instrumentales se asocian positivamente a los esfuerzos por generar "una otra forma» de entender el proceso educativo escolar, particularmente en el contexto del reconocimiento político y social de los derechos de los pueblos indígenas a una educación basada en sus referentes culturales, lingüísticos y formas de organización social y política propias.

Palabras clave: participación comunitaria y social en educación; pueblos indígenas; educación comunitaria; política educativa; innovación educativa.

\section{SUMMARY}

Social participation in education includes a wide field of experiences and approaches. With this article, we want to contribute to this debate from the point of view of the specific experiences from community middle schools in Mexico where participation goes beyond instrumental forms of understanding knowledge and of family support in schools, on the one hand and on the other we discuss schoolcommunity relations as a basis on which to construct new understandings of scholar education, its pedagogy and learning processes. We maintain that active, not instrumental forms of social participation in education are associated positively to efforts to generate different ways of understanding educational processes in schools, particularly, in the context of the political and social recognition of the rights of indigenous peoples to an education based on their cultural, linguistic referents and their own forms of social and political organization.

Key words: social and community participation in education; indigenous people; community education; educational policy; innovative education.

\section{SOMMAIRE}

La participation sociale en milieu éducatif est un terrain riche en termes d'expériences et de perspectives. Dans cet article, nous contribuons au débat depuis une expérience éducative spécifique au sein d'écoles secondaires communautaires au Mexique. D'un côté, cette participation va au-delà des formes instrumentales des connaissances et du soutien familial à l'intérieur des écoles, et d'un autre côté, nous argumentons que la relation école-communauté en tant qu'axe à partir duquel d'autres façons de comprendre l'éducation scolarisée, ses pédagogies et ses savoirs peuvent être encouragées. Nous soutenons que des formes actives de participation sociale en éducation ainsi que d'autres non-instrumentales sont associées positivement aux efforts déployés pour générer "une autre façon" de comprendre les processus d'éducation scolaire. En particulier, dans le contexte de la reconnaissance politique et sociale des droits des peuples autochtones à une éducation fondée sur leurs référents culturels, linguistiques et sur les formes d'organisation sociale et politique qui leur sont propres. 
Mots clés: participation communautaire et sociale en éducation; peuples autochtones; éducation communautaire; politique éducative; innovation éducative.

\section{INTRODUCCIÓN}

El artículo que aquí presentamos centra su atención en las formas de participación comunitaria en educación que, tomando como caso una experiencia específica, reflexiona sobre sus formas democráticas, situadas y dialógicas de participación frente a prácticas que reproducen un enfoque instrumental, no colaborativo ni transformador de la experiencia educativa. Bajo un enfoque antropológico y con herramientas clásicas de los estudios etnográficos -como son el trabajo de campo prolongado, las entrevistas y la observación participante- nos acercamos a esta problemática eligiendo esta opción metodológica por su potencial de «interpretación cultural» (Wolcott, 1993, 128). Para nuestro caso de interés, compatibilizamos una etnografía escolar (cfr. Bertely, 2000; Velasco y Díaz de Rada, 1997) con una etnografía comunitaria en el marco de una etnografía reflexiva (Dietz, 2003) ${ }^{1}$.

Nos insertamos en un vasto campo de conocimiento. Dentro de él, pretendemos hacer dialogar los enfoques de la participación social en educación con perspectivas educativas interculturales en regiones indígenas (cfr. Dietz, 2003; Dietz y Mateos, 2011; Walsh, 2012, 2013; Bertely, 2015; Schmelkes, 2003; Jiménez Naranjo, 2012; Maldonado, 2000; Baronnet, 2012; Sartorello, 2009; González Apodaca, 2006, por mencionar algunos). Nos interesa debatir sobre formas de participación social en lo educativo que rebasen el preeminente afán de comprender el conocimiento comunitario y el apoyo familiar en la educación escolar desde una mirada instrumental. En segundo lugar, pretendemos explorar en qué sentidos la relación escuela-comunidad podría fomentar otro tipo de educación escolarizada, pedagogías y aprendizajes.

Ubicamos ambos intereses en: a) los derechos de los pueblos indígenas a una educación "propia», reconocidos por el Convenio 169 de la OIT, fomentada por procesos de mayor autonomía, la «justicia curricular» (Torres, 2010) y el pluralismo epistemológico (Olivé et al., 2009); b) en las estructurales y desiguales condiciones socioeconómicas que siguen afectando a los pueblos indígenas y la mayor parte de la sociedad nacional; y c) en la emergencia de otras formas de "hacer escuela» (Rockwell, 2007) y la necesaria transformación de la institución escolar en un contexto de «erosión» (Pérez Gómez, 1995) de las formas tradicionales asociadas a ella.

El modelo de las Escuelas Secundarias Comunitarias Indígenas de Oaxaca en México representa una experiencia de apropiación étnica (González Apodaca,

1. El trabajo de campo de este estudio es parte de la investigación "Las secundarias comunitarias indígenas de Oaxaca-resignificaciones de la educación escolarizada desde una propuesta alternativa para la formación de los jóvenes» (Kreisel, 2017), desarrollada en el Instituto de Investigaciones en Educación de la Universidad Veracruzana, México. 
2006) de la institución escolar, la cual apuesta por un proyecto pedagógico diferencial. Entre sus principios filosóficos y epistémicos fundacionales se encuentran la comunalidad como perspectiva teórico-filosófica y forma de vida de los pueblos originarios (Maldonado, 2002; Martínez Luna, 2013; Díaz Gómez, 2007) y el enfoque intercultural. Desde una mirada crítica, se plantea la necesidad de enfrentar los efectos de los procesos históricos de asimilación cultural que han conllevado, como estrategia de integración nacional, la escolarización de los pueblos indígenas de México, que, como constata Esteva $(2014,40)$, ha buscado "des-indianizar a los indios, es decir, de disolver sus culturas e incorporarlos, mediante la escuela, a las formas y normas culturales de la nación".

En el contexto mexicano en general y, muy particularmente, en el oaxaqueño, se han producido numerosos estudios que relatan y denuncian las consecuencias de esta política pública que, con sus diferencias, aún no cesa (De la Peña, 2002; González Apodaca y Cornelio, 2013; Jiménez Naranjo, 2009). Inmersa en este debate, la propuesta que analizamos pretende generar, con una metodología propia, procesos de reivindicación de los saberes originarios y fortalecer las identidades étnicas. Para este fin, se enfoca en la vinculación activa escuela-comunidad, a partir del trabajo por proyectos, en los cuales se busca la articulación de los saberes y la participación de los diferentes actores comunitarios en las actividades educativas.

Dada la "envergadura" del proceso al que aspira, tanto en términos de transformación escolar como de participación, la experiencia se desarrolla en medio de una serie de tensiones en torno a los significados atribuidos a la formación escolar, a los contenidos, a los actores participantes en los procesos escolares, a los tiempos y los espacios del aprendizaje, entre otras dimensiones. El enfrentamiento de una diversidad de retos ha contribuido a generar aprendizajes colectivos sobre una educación escolar participativa y vinculada a la comunidad. Desde nuestro punto de vista, estos aprendizajes aportan a la reflexión sobre la participación social en educación y la transformación de la institución escolar, haciéndola más situada, plural e innovadora. Especialmente, para comprender el aprendizaje en las actuales sociedades del conocimiento y para coadyuvar en la participación social en educación en el marco de los derechos y la autonomía reconocidos para los pueblos indígenas.

\section{CONVERGENCIA ENTRE POLÍTICAS EDUCATIVAS Y ECONÓMICAS}

Estamos habituados a un discurso educativo que enfatiza la formación de niños, niñas, adolescentes y jóvenes como futuros trabajadores y profesionales en competencias que les permitan aportar al progreso del país, en un contexto social, cultural y económico que no únicamente ha de comprenderse como nacional, sino que es pensado en términos de las exigencias de la globalización y de la sociedad postindustrial. En este marco, la gestión del conocimiento se ha convertido en un tema central y ha adquirido también tonalidades instrumentalistas, tecnócratas y administrativas (Treviño, 2015). 
La participación social en educación -como política pública en Méxicoabreva de un conjunto de políticas económicas y educativas globales, a las cuales responde. Observamos convergencias, en el ámbito latinoamericano, entre políticas educativas que acentúan el interés por la calidad educativa, eficiencia, competitividad y evaluación y tendencias económicas que ahondan el adelgazamiento del gasto y responsabilidad social de los Estados, fragmentación social y empobrecimiento acelerado (Pedró y Rolo, 1998, 264).

Calidad, eficiencia, equidad y participación social en educación son principios que rigen la mayor parte de las reformas educativas en estos contextos, inspiradas y alimentadas muchas veces por los lineamientos que se elaboran desde los grandes centros de poder financiero (Gigante, 2003, 64); por los compromisos que se establecen a través de los acuerdos multilaterales regionales como el Tratado de Libre Comercio (TLC), mercosur o el Pacto Andino, y por las agencias de cooperación de distintos países que operan en Latinoamérica (Gigante, 2003, 66). Los focos de poder económico y de crédito, de esta manera, contribuyen a que se lleven a la práctica políticas y estrategias que conducen a una convergencia educativa escolar en el continente (Pedró y Rolo, 1998, 269).

Pero también organismos internacionales como el Banco Mundial (BM) o el Fondo Monetario Internacional (FMI), o la Organización de las Naciones Unidas para la Educación, la Ciencia y la Cultura (UNESCO) y la Organización para la Cooperación y el Desarrollo Económicos (OCDE) respaldan propuestas de mayor participación social en educación, sostenidos en los idearios de las nuevas sociedades de la información o conocimiento (cfr. Treviño, 2015). Son compromisos de orden global que México se compromete a cumplir, de los cuales abreva y a los cuales rinde cuentas. En conjunto, se asiste a políticas económicas de apertura, eficiencia, reducción del gasto y responsabilidad social de los Estados, flexibilización del mercado laboral y reformas educativas que acentúan la relación entre calidad educativa, eficiencia, competitividad, evaluación y participación.

Mientras más profundos son los procesos que vinculan la flexibilización y competitividad económica con la reducción del gasto en educación más aumentan las necesidades financieras que vinculan eficiencia en la gestión y el gasto educativo con la participación social de padres y madres en el área educativa. Esta situación alimenta políticas educativas que, en cuanto al aprendizaje, toman como centro de interés la evaluación y la eficiencia del conocimiento y vinculan muy estrechamente el aprendizaje de niños, niñas y jóvenes para adaptarse eficazmente a un mundo laboral cambiante (OEI, 1998, 253).

Es interés de este trabajo aportar conocimientos teóricos, analíticos y empíricos que permitan discutir que el impulso de la participación social en educación desde una visión instrumental -en la que ahondamos en lo que sigue-favorece una visión compensatoria de los desajustes provocados por las políticas económicas y pretende amortiguar los efectos de políticas educativas globales de flexibilización y competitividad del mercado educativo escolar. Por contraparte, y alejándonos de esta visión compensatoria e instrumental de la participación social, aportaremos 
YOLANDA JIMÉNEZ NARANJO Y MAIKE KREISEL

PARTICIPACIÓN COMUNITARIA EN EDUCACIÓN-RECONFIGURACIONES DE LO ESCOLAR. .

elementos analíticos y empíricos que permitan observar la participación social en educación indígena desde posiciones más transformadoras, tanto del sentido de lo escolar y del aprendizaje, como de los derechos de los pueblos indígenas a una educación más apropiada a sus fines políticos, sociales y económicos.

En este marco, nos interesa observar cómo el papel de la participación social en educación se puede rearticular desde una dimensión de democratización y participación colectiva en el ámbito educativo escolar y, en un segundo momento, aventurar las transformaciones que trae parejas sobre la forma de entender el aprendizaje y el conocimiento. Consideramos que, en términos generales, lo expuesto en el presente análisis permite reflexionar también otros espacios educativos no ligados al mundo indígena. Sin embargo, en este artículo tomamos como caso específico un modelo que liga su experiencia a los derechos educativos de los pueblos indígenas y a la necesidad de transformar el sentido del aprendizaje escolar. Su caso nos parece muy significativo para los propósitos que queremos señalar sobre la visión instrumental en participación en educación y la necesaria superación de este enfoque.

La experiencia se enmarca en procesos más amplios de apropiación y reinvención de la institución escolar que forman parte de la lucha por el reconocimiento de los derechos indígenas en México, pero también en otras regiones de América Latina. La apropiación escolar desde posicionamientos etnopolíticos (González Apodaca, 2006; Bertely, 2015) ha implicado la negociación, a veces abierta, pero frecuentemente también tácita, de las relaciones de poder que implican el control de los espacios educativos en cuestión (Rockwell, 2005). Estos se convierten así en lugares de identidad, apuntando hacia el diseño de proyectos de futuro comunitario, alejados de los que prevén las políticas oficiales. Es en este contexto que queremos profundizar en procesos de participación social en educación no instrumentales, es decir, de mayor autonomía, reapropiación e innovación sobre las clásicas prácticas educativas.

Si bien encontramos procesos de ruptura, la sedimentación de esas prácticas más tradicionales en el imaginario y en las formas cotidianas de "hacer escuela" juega como un poderoso retractor del cambio y de las posibilidades del modelo. Aun así, su esfuerzo nos permite dialogar sobre la activación, caminos, resistencias y alcances de prácticas de mayor participación comunitaria en educación y sus transformaciones sociopedagógicas.

3. PARTICIPACIÓN SOCIAL EN EDUCACIÓN: DESDE LA INSTRUMENTALIZACIÓN HACIA PROCESOS CO-CONSTRUCTIVOS

El Acuerdo Nacional para la Modernización de la Educación Básica (ANMEB) y la Reforma Educativa de 1993 en México son referentes claros en los cuales la participación social en educación se formaliza y oficializa; en la misma Ley General de Educación, reformada en 1993, quedan asentados diferentes órganos de participación en educación (Estrada, 2014, 733). Desde entonces, los Consejos Escolares 
de Participación Social (CEPS) han sido un instrumento principal en la forma de entender e impulsar la participación social en el contexto educativo (Zurita, 2011, 143) desde el ámbito de la política pública. El campo de conocimiento que abarca la participación social en educación, tomando como eje el funcionamiento de los CEPS, es vasto. Si bien la normatividad asociada a ellos fomenta la participación desde diferentes ejercicios, su implementación ha estado sujeta a tensiones en la práctica.

Algunas de las críticas a la participación escolar de los padres y madres de familia señalan que esta suele concentrarse en el pago de cuotas y el mantenimiento de las instalaciones (Flores y Ramírez, 2015, 80). Persiste «una separación histórica escuela-familia y una serie de desencuentros y desconfianzas» (Estrada, 2014, 737). En el desempeño práctico, los CEPs han enfrentado una serie de dificultades, entre ellas, una falta de información sobre sus objetivos, una visión más bien instrumental, además de la poca claridad en cuanto a la diferencia de las funciones de la Asociación de Padres de Familia y los mismos Consejos y en regiones indígenas, afecta la falta de contextualización de los mismos CEPS (Zurita, 2011, 144). Perales y Escobedo (2016, 78-79), en esta sintonía, sugieren "reconsiderar las instancias que promueven la participación social», adaptándolas a la vida cotidiana de los actores locales y sus formas sociales y culturales de organización, además de entender la participación social en educación como parte del ejercicio de y la formación para una ciudadanía más democrática, de mayor equidad, autonomía y transparencia, contribuyendo a condiciones sociales más justas.

Lo que los autores concluyen es extensivo también para las escuelas regidas bajo el manto normativo de la Dirección General de Educación Indígena (DGEI). En una evaluación nacional sobre la política pública relativa a la DGEI, las autoras Jiménez Naranjo y Mendoza (2012, 119 y ss.) constatan que la participación de padres y madres de familia se relaciona fundamentalmente con la recaudación de fondos económicos que subsanan deficiencias de diversa índole (que gestionan y gastan los docentes), tales como construir aulas nuevas o comprar material. Muy notoria es también su participación en cuestiones de limpieza. Esto es característico en regiones rurales e indígenas donde las escuelas no cuentan con presupuesto para intendente y son las familias o niños y niñas quienes asumen estas actividades. En este sentido, la participación social en calidad de "mano de obra» es recurrente para la construcción de aulas, gestión de recursos, en colaboración en diferentes eventos escolares y extraescolares, así como la práctica de compartir con los padres de familia algunas funciones de "vigilancia». En una labor de supervisión, también las familias son llamadas a cuidar el cumplimiento de las obligaciones de estudiantes y docentes, así como de la eficiencia y transparencia del gasto.

En conjunto, puede decirse que las actividades de participación de las familias en educación se concentran en un papel extraeducativo, como proveedoras de recursos y mano de obra, controlado y dirigido por las necesidades que docentes e institución educativa plantean -y no al revés- y, recientemente, en el marco de 
la institución de los CEPS, de vigilancia y control del gasto ${ }^{2}$. Por otro lado, cuando se les solicita participación educativa, esta se concentra en "ayuda en casa" para conseguir los aprendizajes esperados que docentes e institución escolar deben propiciar en niños y niñas.

En general, la participación de las familias en las escuelas, articulada a través de las vías institucionalmente instituidas -y aunque muy activa-, comparte esta visión instrumental, suple funciones presupuestales que corresponden al Estado y colabora en muy poco en la necesaria transformación de los sentidos actuales del aprendizaje y de la democratización de la vida escolar. Sin este tipo de participación -que es la mayoritaria-, el proyecto de educación pública no es posible en Estados que, como el mexicano, proclama la llegada e implementación de una escuela de calidad en un contexto de retirada y adelgazamiento del gasto público en educación. Se suma a esto una precarización y desigualdad del presupuesto en regiones históricamente marginadas. Por ello, la participación social en regiones indígenas -en los términos antes descritos- se ha vuelto cada vez más relevante para el erario público, y especialmente bajo el planteamiento de una nueva "autonomía de gestión» escolar (Rivera, 2016), introducida con la reforma educativa del año 2013 en México.

Se trata, a nuestro juicio, de formas muy limitadas de entender la participación, puesto que ahondan en un sentido instrumental y funcional a la reproducción de un sistema educativo que tiende a su reducción en gasto público, que plantea ampliar el poder socializador y sus mecanismos de conocimiento hacia las familias o comunidad indígena y no al revés. Es decir, debería, al contrario y al menos en regiones indígenas, tratarse más bien de una participación que resalte el papel de las familias como sujetos activos y propositivos en el aprendizaje de sus hijos e hijas y en las definiciones escolares, vinculando el conocimiento comunitario y sus epistemes con otros desde un punto de vista dialógico y plural o considerando que la participación en la definición de las prácticas educativas es parte fundamental del ejercicio del derecho a la autonomía que diversos documentos normativos al interior del país reconocen para los pueblos indígenas.

\section{POLÍTICAS EDUCATIVAS Y LA RESIGNIFICACIÓN CURRICULAR: APROPIACIONES ÉTNICAS DE LA ESCUELA}

Rockwell y Ezpeleta $(1983,1)$ afirman, a partir del análisis de políticas y reformas educativas, que en el sistema educativo mexicano se proyecta una escuela que en su concreción "no es", pues es mirada desde arriba y definida desde categorías

2. No obstante, hay que mencionar también el análisis realizado por Elsie RockwelL (2005) de las dinámicas de apropiación escolar ejercidas por las comunidades y los padres de familia de los procesos de la introducción de la escuela pública en el ámbito rural, que desde entonces implicó formas alternas de vigilancia y control, apuntando a la historicidad de las tensiones de poder entre institución escolar y actores comunitarios. 
concebidas desde estructuras de poder. La noción subyacente del currículum ha sido basada en "una concepción administrativa: regular y controlar las prácticas educativas que tienen lugar en el sistema educativo en general, en los centros y aulas en particular» (Bolívar, 1999, 151). Díaz Barriga $(2012,26)$ enfatiza que en el contexto mexicano esta persistente verticalidad se articula también en los cambios educativos y las reformas. Son implementados verticalmente como innovaciones concebidas desde el Estado, excluyendo a los demás actores sociales de un espacio público y de su renovación. La participación social en este sentido también se ha instrumentalizado, por ejemplo, a través de consultas en regiones indígenas para legitimar las reformas implementadas (Jiménez Naranjo y Mendoza, 2016), inhibiendo procesos de participación ampliada y de democratización de la educación escolarizada.

No obstante, creemos que, para comprender la vida de las escuelas, habría que partir no de una visión estática y vertical, sino de su construcción social, local y particular, desde los sujetos y sus interrelaciones, desde procesos históricos situados. La historia de la escuela homogénea y homogeneizante, concebida desde las estructuras macro, coexiste con las otras historias, de la sociedad civil, de estudiantes, de las familias y cómo estos realizan sus apropiaciones y recreaciones del espacio escolar (Rockwell y Ezpeleta, 1983, 3)³. Más allá de las políticas gubernamentales se trataría de comprender a la "escuela" también como una construcción social y cotidiana. En estas resignificaciones concretas se rearticulan las relaciones de poder, se presentan tanto reproducciones como resistencias. El currículum escolar, de esta forma, es «deconstruido a través de sus agentes sustantivos» (Paraskeva, 2001, s/p): estudiantes, maestros y familias, generando reconfiguraciones de manera multiactoral. Estos procesos no se encuentran exentos de tensiones a nivel local, en los cuales se tejen también procesos e interrelaciones de poder.

Como hemos mencionado, existen en México, pero también en otros lugares de América Latina, experiencias de reconfiguración y apropiación escolar que, en algunos casos, han sido promovidas explícitamente en el marco de iniciativas y movimientos etnopolíticos por actores sociales locales en diferentes alianzas. Se han generado propuestas educativas diferenciales, de corte comunitario, que se han constituido como un campo importante de innovación en la construcción de escuelas y pedagogías distintas. En el marco de las reivindicaciones étnicas, el derecho a la creación de modelos educativos diferentes a las propuestas nacionales se fomenta en los derechos colectivos de los pueblos indígenas y se dinamiza desde la demanda emergente y lucha política por ejercer la ciudadanía diferencial, o étnica (De la Peña, 1995).

En el estado mexicano de Oaxaca, en el que se inserta la experiencia educativa a la que hacemos referencia, la defensa de una educación propia ha sido impulsada por el movimiento magisterial indígena, pero también por las mismas comunidades

3. En este sentido, para el caso español, por ejemplo, nos parecen interesantes el caso de las comunidades de aprendizaje y algunas de sus apuestas (BARRIO, 2005). 
e intelectuales indígenas, actores institucionales y académicos. Para ello, han sido claves los movimientos sociales de las recientes décadas, entre los que destaca el emblemático levantamiento zapatista, pero a ellos subyace también el mismo movimiento estudiantil del 68 como inicio de una nueva conciencia y protestas de la sociedad civil (Favela, 2008, 92). Estas transformaciones sociales se han traducido en una reetnificación de las demandas de los pueblos originarios, donde «la etnicidad y no la ciudadanía definida de forma individualista y formal se convierte en principal fuente y objeto de las reivindicaciones" (Dietz y Mateos, 2011, 82).

La concepción de derechos diferenciales, según Leyva (2007, 37), pone «en jaque la noción liberal de democracia, igualdad y ciudadanía sobre la cual se había erigido el Estado mexicano». En este marco, Bertely (2007, 21) busca esclarecer "Cómo hacer efectiva una ciudadanía diferenciada en condiciones de igualdad", haciendo especial énfasis en las escuelas como espacios en torno a los cuales se disputa el ejercicio de los derechos. Se configuran como «arenas de poder en que tienen lugar las apropiaciones étnicas» (González Apodaca y Rojas, 2013, 282). Para el caso de nuestro interés, nos aproximamos al espacio escolar como territorio en negociación, en tensión profundamente relacional donde confluyen procesos de construcción de posiciones de los grupos dominantes y dominados, a través de los enfoques epistemológicos en disputa (Silva, 1999). En México, bajo esta perspectiva, a pesar de reformas que conciben la flexibilización del currículum, podemos observar cómo, frente a las emergencias de propuestas innovadoras que cuestionan las concepciones clásicas de "lo escolar», las estrategias políticas actuales restablecen dinámicas centralizadas en cuanto a administración y evaluación, y los planes y programas oficiales siguen concibiéndose desde lógicas jerárquicas.

\section{El modelo de las Secundarias Comunitarias Indígenas de OAXaCA: Formas ALTERNATIVAS DE EDUCACIÓN ESCOLAR}

El modelo de las Secundarias Comunitarias Indígenas (ESCI) de Oaxaca nació, hace catorce años, en el seno del Movimiento Pedagógico, promovido por la Coalición de los Maestros y Promotores Indígenas de Oaxaca (CMPIO), que forma parte del movimiento magisterial democrático de México. Ha buscado innovar los aspectos pedagógicos para una educación pertinente para las comunidades, apostando por el involucramiento directo de los actores locales y la comunicación con los padres de familia. Como fruto de las iniciativas de la CMPIO, el modelo de las Secundarias Comunitarias Indígenas se inserta en el panorama de la oferta educativa estatal oficial. Desde el año 2004 comenzó a operar, a partir de un acuerdo entre la Sección XXII y el Instituto Estatal de Educación Pública de Oaxaca (IEEPO), con la acreditación oficial de los estudios. Actualmente, se encuentra sujeto a tensiones y procesos de negociación política, entre el reconocimiento de un proyecto piloto e innovador y la falta de apoyos financieros y humanos para su operación.

Las Secundarias Comunitarias, con diez planteles insertos en comunidades de diferentes regiones del estado, han recorrido ya un trecho del camino de la 
concreción práctica del modelo, rearticulando, a partir de la experiencia, algunos aspectos de su metodología y ajustando su funcionamiento de acuerdo a los aprendizajes obtenidos. Se ha impulsado un proceso de formación docente continuo y en diálogo con diferentes actores institucionales, académicos y comunitarios, para seguir consolidando el trabajo pedagógico. Dicho proceso ha contribuido a interesantes y sugerentes procesos de apropiación y resignificación del modelo por los actores educativos y comunitarios (cfr. Ruiz y Quiroz, 2014; Briseño, 2015; Kreisel, 2016a).

En lo que sigue pasaremos a exponer dos pilares fundamentales del modelo y relevantes para la comprensión de las premisas que sostiene este artículo.

\subsection{El trabajo por proyectos; más allá de la gramática escolar}

El currículum de las secundarias comunitarias se desarrolla de acuerdo a una metodología basada en proyectos de aprendizaje y de investigación. Con cada nueva generación de estudiantes, las temáticas a estudiar se construyen, dialogan y negocian con docentes y habitantes de la comunidad. Existe, frente a una lógica vertical y cerrada del currículum nacional -cabría decir además no situada ni pertinente a las necesidades locales-, una construcción permanente y continuamente innovada por los participantes. Las temáticas de los proyectos son elegidas por el colectivo de actores, integrado por padres de familia, estudiantes, Consejo Representativo -como órgano que reemplaza en el modelo al comité de padres de familia, pretendiendo incidir en nuevas lógicas de participación- y educadores. A través de la metodología propia, los temas seleccionados determinan, de manera flexible y partiendo de intereses y necesidades de relevancia comunitaria, qué prácticas, conocimientos o problemáticas se abordarán a lo largo del ciclo escolar.

Nosotros seguimos apostándole a un currículo de construcción permanente en donde se van revisando distintos escenarios en cada generación, aun en la misma escuela [...]. La idea es estar siempre en un proceso y direccionarlo a como se vaya direccionando la realidad [...] (E-asesor pedagógico).

No se trabaja por asignaturas, sino que el mismo proyecto, organizado a través de un esquema de investigación y cuyas actividades se planean de manera conjunta, pretende generar un diálogo entre conocimientos comunitarios y conocimientos procedentes de las ciencias, de otros contextos culturales, etc. Rompe con ello con dos presupuestos fundamentales asociados a lo escolar; la eliminación de asignaturas como tal -porque el aprendizaje y el conocimiento se entienden holísticos- y el posicionamiento crítico que asume frente al currículum nacional oficial. Si bien trabaja la articulación de los conocimientos comunitarios con los escolares o científicos, esto se hace a partir de las necesidades localmente sentidas y de las

4. Usamos gramática escolar en el sentido que lo realizan TYACK y CUBAN, 1995. 
necesidades que el aprendizaje autónomo de jóvenes demanda, el cual está más encaminado a la formación en investigación que a la adquisición de contenidos descontextualizados.

Todos los contenidos que vamos a trabajar también los dialogamos con los papás, con todos los actores que están involucrados. Que no es un ejercicio sencillo, lleva su tiempo y tiene sus implicaciones. Entonces por eso, por ejemplo, en este caso, en el caso de la secundaria, no nos conflictuamos con el asunto del contenido. Porque la misma metodología nos va a llevar a construir el esquema de lo que vamos a trabajar (Transcripción-integrante coord.-taller DEI).

Además de abordar problemáticas locales detectadas por estudiantes y comuneros, el modelo busca "recuperar» el saber que se entreteje en la memoria histórica de la comunidad, conservado y tradicionalmente transmitido por los mayores, quienes son considerados, en las ESCI, Como "portadores de conocimiento". Estos conocimientos son percibidos como amenazados o ya en proceso de desplazamiento, a causa de los procesos de aculturación vinculados a los rápidos avances de la tecnología y el acceso a la información, además del consumo, cada vez más presentes en el territorio comunitario a través de los medios, diversos artefactos y productos. La misma escuela, como instrumento y vía de la homogeneización cultural promovida por el Estado-nación, ha sido parte de ello.

El posicionamiento reivindicativo busca revertir estas lógicas sin excluir el diálogo; «lo que nosotros planteamos es un diálogo cultural, de carácter horizontal, en donde el conocimiento comunitario sea la guía para entrar al otro conocimiento, el conocimiento occidental, que no lo negamos, sino que entramos ahí a través del propio» (E-asesor pedagógico). Para poder partir del conocimiento comunal, la escuela se abre hacia la vida cotidiana de la comunidad. Los proyectos de investigación giran en torno a la investigación de las prácticas productivas -situadas, por ejemplo, en la milpa, el cafetal o el cañaveral-; de las formas de vida locales, reflejadas en la misma construcción de las casas, en la medicina tradicional, entre otros; pero también de las problemáticas que se enfrentan, como pueden ser el alcoholismo, la pérdida del respeto, la contaminación y deforestación, para mencionar solo algunos de los aspectos que han sido profundizados en los diferentes planteles. Los temas son definidos en los «seminarios de apertura» que involucran a padres y madres, estudiantes y educadores. El conocimiento en torno a estos tópicos solo es posible de construirse a través de una relación dialógica con el contexto y sus actores, a través de la permeabilidad de escuela y currículum a su entorno, y son complementados con investigaciones bibliográficas, retomando elementos del currículum de la Secretaría de Educación.

En el modelo hay inserto un cuestionamiento sobre las clásicas formas de adquirir conocimiento escolar: repetición, memorización, aprendizaje dirigido y no significativo y las relaciones autoritarias y verticales de docentes hacia estudiantes. Muy al contrario, se fomenta el aprendizaje autónomo, colaborativo y autorresponsable de los y las jóvenes. El educador, a través de un proceso de formación 
continuo, aprehende dinámicas para fungir como guía en el proceso de autonomía y autorresponsabilidad del o de la estudiante en su aprendizaje. Se trata de un aprendizaje situado y autorregulado, que busca tener relevancia local y que es construido con los demás habitantes de la comunidad. Estas problemáticas, al ser "llevadas» al aula ${ }^{5}$, se inscriben también en las lógicas académicas o escolares; los tópicos comunitarios son sistematizados y ampliados bajo una lógica de investigación, son comunicados por escrito o verbalmente en un lenguaje apoyado en lo académico (aunque de forma bilingüe) ${ }^{6}$. Se impulsa su comparación y análisis en relación a otras problemáticas nacionales o globales. Asimismo, la lógica de investigación implementada fortalece el conocimiento científico en tanto busca conocer, de manera sistemática bajo la metodología implementada por el modelo, las respuestas que el conocimiento científico ofrece a las preguntas que interesan a la comunidad y a los y las jóvenes. Por ello no es un conocimiento que se niegue, pero se accede a él desde un lugar distinto al convencional.

\subsection{Las distintas dimensiones de la participación}

La participación atraviesa todo el modelo. Es difícil analizarla de manera aislada, puesto que, como hemos visto, está inserta en la misma metodología de proyectos, en las aproximaciones pedagógicas, además de articular también las formas organizativas. Quisiéramos, no obstante, en este apartado, abordar los espacios en los cuales la participación se construye como experiencia explícita.

I) El Consejo Representativo. Como hemos mencionado, es una figura propia del modelo que busca replantear conscientemente el papel tradicional de los "comités de padres de familia" que ha marcado la forma de interacción más instrumental en las comunidades. El Consejo es constituido a partir de las dinámicas de organización propias de la comunidad -la asamblea comunal y la asignación de cargos por esta- y pretende involucrar a los ciudadanos participantes también en los procesos propios de la investigación en cada proyecto. Constituye, además, un vínculo importante para los procesos de apropiación local del modelo a través de la participación de sus integrantes en la escuela y su familiarización con sus formas particulares de trabajo educativo. Participa en los procesos de toma de decisión sobre asuntos escolares, en conjunto con los educadores y en comunicación con los demás actores.

5. Sin pretender ser extensos en este punto, también reconocemos que en algunas ocasiones estas prácticas se ven limitadas a la introducción de elementos aislados y cosificados, frecuentemente folklorizados, como "Contenido cultural» al aula (JimÉnEZ NARANJO, 2009).

6. El diálogo entre lenguas que se desarrolla en este proceso encierra sus propias complejidades, también epistemológicas, en las que aquí no profundizaremos. 
II) La construcción conjunta de los proyectos. Familias, comuneros, estudiantes y docentes participan en la definición de las problemáticas que van a abordar los proyectos -como acabamos de ver-, en los seminarios de apertura. Además, es ante las familias, el Consejo Representativo y, eventualmente, las autoridades comunitarias que los y las estudiantes presentan periódicamente sus avances de investigación. Estos espacios forman parte de la evaluación, y en ella se observan tanto los aportes de las investigaciones de los y las estudiantes como las capacidades argumentativas y de exposición. Se espera que los hallazgos de cada proyecto logren no solo recuperar y registrar saberes comunitarios, sino también contribuir a la reflexión conjunta sobre problemáticas locales y sus posibles soluciones, además de trascender, a través de los proyectos de investigación de tercer grado de secundaria, con alguna iniciativa práctica de impacto comunitario.

III) El proceso de construcción de conocimientos. La dinámica del trabajo por proyectos y la metodología implementada ahonda en el conocimiento local como uno que es válido y validado para ser tema de estudio, y los comuneros, como "portadores de conocimiento comunitario", se convierten en quienes aportan, a través de su saber y experiencia, a los procesos educativos y reflexivos de la formación de los jóvenes, vinculando escuela y comunidad. Así se observa, por ejemplo, cómo se desarrolla el diálogo entre jóvenes y comuneros sobre la contaminación en la comunidad cuando seis ancianos, hombres y mujeres, fueron invitados a participar en la escuela en una "conferencia de ancianos", en la que se abordaron memoria histórica y apreciaciones críticas en torno al tema.

Se habló de cuentos que se han transmitido, del pasado, se habló de las condiciones de vida, desde antes de la revolución, de que la vida antes de la llegada de la carretera era muy dura, había libertad y soltura, pero también sufrimiento, mucho sufrimiento; que la carretera había traído tanto beneficio como sacrificio (esto lo decía Don Moderado), ventajas y desventajas. Los avances tecnológicos fueron otro tema, la televisión encontró bastante rechazo por parte de los ancianos, tanto como también los celulares; se habló de la misma manera de la comida chatarra, y los gastos que todo ello generaba, a la vez de perjuicios para la salud. Se habló de los químicos que se les ponen a las frutas compradas, en comparación con las locales, pero que ya les daba a veces flojera de ir por las de la comunidad, y que las naranjas compradas eran bañadas en químicos. [...] Los alumnos, con cierta timidez, plantearon las preguntas que habían previamente organizado junto con [el educador] [El educador] intervino y moderó en zapoteco, a pesar de la diferencia regional. (Obconferencia de ancianos-3. ${ }^{\text {er }}$ grado).

IV)La interlocución entre educadores y comunidad. Los equipos de educadores comunitarios que desempeñan su función como mediadores de los 
procesos formativos en cada plantel son concebidos como actores centrales en el proceso dialógico entre escuela y comunidad, convirtiéndose en aprendices e investigadores respetuosos del saber local y contribuyendo a la resignificación conjunta, siempre negociada, del papel de la institución escolar como espacio comunitario (Kreisel, 2016b).

V) Para ello, las formas comunitarias de decisión y lógicas culturales y sociales son puestas en interacción respetuosa y dialógica, articulándose entre escuela, educadores, consejo y asamblea comunitaria, autoridades y cabildo, entre otras instancias y dimensiones.

Los alcances de la participación de los diferentes actores en la educación escolar se articulan, más allá de ello, en estos u otros espacios -menos explícitos, intersticiales-, en las dinámicas de interacción que se logran impulsar en la práctica y en las que se presentan diferentes grados de efectiva interlocución, construcción y toma de decisiones conjunta.

\section{LOS ESPACIOS DE TENSIÓN; INTERSTICIOS DE LA PARTICIPACIÓN}

La relevancia de estas formas extendidas y ampliadas de participación en educación que muestra el modelo de las Esci no ocurren sin desacuerdos, negociaciones y rupturas. Estas no podrían estar exentas de negociaciones de poder; no siempre son simétricas, pero sí se constituyen en la búsqueda de la horizontalidad y en la necesaria capacidad de "la escuela» de, en lugar de aislar e imponerse, concebirse como construcción conjunta que, solo a partir de la dimensión colectiva y comunitaria del quehacer educativo, puede cobrar sentido y pertinencia. Esto es lo relevante a nuestro juicio; reconocer los importantes avances en los procesos de apropiación del modelo por parte de las comunidades -en ya 13 años de implementación-, pero sin negar estas tensiones y diferencias. Al contrario, ha de celebrarse que se inscriban en un ejercicio democrático, dialógico, plural e incluyente dentro de una práctica educativa escolar.

Son, por tanto, tensiones que forman parte del mismo proceso dinámico e inconcluso, pero reflexivo, autoapropiado y participativo, que defendemos que debe existir en el ámbito de la participación escolar no instrumental. Sin poder ahondar en estas tensiones en este trabajo - por la breve extensión de un artículo, aunque podrán encontrar más ejemplos empíricos y voces directas de sus actores en el trabajo en extenso de Kreisel (2017)-, nos importa resaltar, aunque sea brevemente, un proceso relevante. Este tiene que ver con la tensión entre un modelo que "experimenta" formas no convencionales de co-construir conocimiento en el ámbito escolar y de extender el propio sentido de participación en esta área, y las expectativas de las propias autoridades educativas estatales -y aún más federales-, pero incluso de algunos padres de familia, quienes insisten en una implementación más vertical de las formas y «haceres» escolares. 
Existen tensiones entre un proyecto que desregulariza las formas tradicionales de "hacer escuela" y la propuesta por una construcción del conocimiento y del aprendizaje situada, de relevancia comunitaria ${ }^{7}$, autónoma y de mayor calado democrático. Especialmente en un contexto en el cual las autoridades educativas no acaban de reconocer y apoyar del todo este quehacer pedagógico innovador y, sobre todo, ante la persistencia de las desigualdades estructurales que afectan a los pueblos indígenas pero también urbanos, donde, aún, las expectativas hacia la educación escolar tradicional alimentan un imaginario -falso y cierto a la vez- de la necesidad de estas formas clásicas de aprendizaje escolar para "triunfar» en la vida económica y salir de la pobreza, en un país de profundas asimetrías sociales y económicas.

Los actores involucrados en el modelo, desde sus diferentes posiciones, han de resignificar sus propias trayectorias e imaginarios, en la mayoría de los casos relacionados con la experiencia de la educación escolar de corte clásico que ha marcado a las comunidades, pero también a la misma formación del magisterio indígena. Es en este marco que una asesora afirma que «el estudiante, cuando llega a la secundaria, está también participando de una biografía escolar y un tipo de biografía escolar distinta de la que venía practicando» (Transcripción-asesoracurso). Los jóvenes, al ingresar al modelo, han tenido que enfrentarse con la necesidad de repensar sus formas de comprender la escuela y asumir un rol activo en ella y en relación a la comunidad.

No, en la primaria pues siempre estábamos encerrados ahí, leyendo libros y la maestra o el maestro nos explica, se ponía a explicar y a explicar, y pues nosotros no hacíamos nada. Y llegamos ahí en la secundaria, al principio, sí, bueno, para mí fue un poquito pesado, salir y andar en la comunidad, pues sí daba flojera. Y pues ya después de un mes, dos meses, ya nos acostumbramos. Y ya sabíamos, de que íbamos a salir o nos llevaban ahí en el campo también. Y ya, y sí fue bonito también porque no todo el tiempo estuvimos encerrados ahí. Y sí nos gustó (E-Zara-egresada-1. ${ }^{\text {era }}$ generación).

Las apreciaciones que insisten en el «regreso» o mayor cercanía a gramáticas escolares tradicionales (Tyack y Cuban, 1995) se relacionan con las percepciones en torno a la falta de oportunidades que los jóvenes de origen comunitario llegan a enfrentar al buscar continuar sus estudios o encontrar, incluso, opciones laborales en el espacio citadino. Incide tanto la experiencia de marginalización que forma parte de la historia de las diferentes generaciones, como también con el contacto cada vez más pronunciado de las comunidades con el contexto social, cultural y

7. No es objeto de este breve artículo entrar en el debate de la relación entre relevancia social y comunitaria y procesos escolares. Sin embargo, aunque sea brevemente, quisiéramos apuntar que el modelo y su metodología, que acerca estos espacios de conocimiento escolar con los conocimientos e intereses locales, son valorados positivamente por sus actores, aunque con tensiones. También los mismos comuneros han llegado a resaltar el carácter ajeno, académico y escolar de las iniciativas de participación que se les han pedido en la escuela tradicional, y las preocupaciones prácticas que, en contraste, ellos tienen que resolver en torno al mundo laboral del campo, que es su medio de subsistencia y vida. 
económico más amplio. Se requiere poder transitar entre espacios, negociar y participar en el contexto local, pero también urbano y nacional.

Sin embargo, tampoco está claro que las clásicas formas escolares sean un medio efectivo para salir de la pobreza o para continuar en el sistema educativo de forma "exitosa». Los indicadores educativos más bien apuntan a la deficiencia de una educación que carece de pertinencia cultural y lingüística en regiones indígenas como explicación del fracaso escolar o de indicadores y experiencias educativas no favorables (González Apodaca y Cornelio, 2013). Además, no solo se trataría de diagnosticar grados de eficiencia o éxito escolar, sino de reivindicar los derechos educativos y culturales de los pueblos indígenas que los cobijan -sin etnocidio, epistemicidio-, sin violaciones a sus procesos de autonomía.

Por eso, la paulatina apropiación de la secundaria por los diferentes actores implica resignificar la institución escolar, y a la vez es un proceso constitutivo para la continuidad del modelo. Como parte de ello, sostenemos que, incluso en términos de éxito escolar y aprendizaje, habría que resaltar como mucho más positivo un modelo educativo que comprenda la construcción de aprendizaje y conocimientos de forma situada, que responda a necesidades social y localmente detectadas y que impulse procesos dialógicos, participativos y autodidactas. Y que estos procesos los promueva sin menoscabo de las identidades, prácticas culturales y lingüísticas de las familias de los niños y niñas que asisten a las escuelas, reforzando de esta manera, en lugar de negar, el aprendizaje significativo y las experiencias de otras realidades y otros conocimientos.

El mismo arraigo comunitario también posibilita la adquisición de otros conocimientos y de otras competencias para la interlocución con la sociedad mayoritaria. Lo hace desde otra manera y desde otro lugar -de mayor respeto-, igualdad, empoderamiento y retomando las lógicas propias de las comunidades. Sin embargo, el valor de esta formación es frecuentemente argumentado como una imposibilidad por los detractores de estos modelos bajo la premisa de que, si una educación vincula a los niños y niñas a la comunidad, perpetúa con ello la desigualdad socioeconómica de la cual son sujetos. No obstante, es ese mismo razonamiento que alimenta visiones instrumentales, verticales, impositivas y etnocidas el que no ha logrado hacer desaparecer, o siquiera reducir, la desigualdad socioeconómica que dice combatir.

\section{CONCLUSIONES}

Este artículo quiere aportar al debate sobre la participación social en educación tomando como caso el modelo educativo de las Secundarias Comunitarias Indígenas, en México, el cual cuenta con 14 años de experiencia. Se trata de un modelo que, en un proceso constante de rearticulación y reapropiación, ha contribuido a un proceso dialógico y participativo entre todos los actores educativos. Está reformulando, en un proceso no libre de tensiones, muchos de los sentidos 
de la educación escolarizada y debatiendo el derecho que otros conocimientos y epistemes deberían tener en espacios como el escolar.

A través de una metodología basada en proyectos de aprendizaje e investigación, las temáticas a estudiar se construyen, dialogan, negocian y evalúan entre docentes, estudiantes y habitantes de la comunidad. Existe, frente a una lógica que es característica del sistema oficial -vertical, cerrada, no situada ni con pertinencia cultural del currículum-, una construcción permanente, dialógica e innovada por los participantes, en la que interactúan, sin perfilarse de manera dicotómica, las necesidades de conocimiento localmente sentidas con los aprendizajes escolares o científicos en un modelo que va más encaminado a la formación en investigación que a la adquisición heterónoma de contenidos escolares descontextualizados. Bajo estos supuestos discutimos que:

a) Formas instrumentales y compensatorias de entender y hacer efectiva la participación social en educación se ligan con tendencias económicas que ahondan el adelgazamiento del gasto y responsabilidad social de los Estados (Pedró y Rolo, 1998, 264).

Es un lugar común en las investigaciones realizadas encontrar que las actividades de participación de las familias en educación se concentran en un papel extraeducativo, es decir, como proveedoras de recursos y mano de obra, que atienden las necesidades que docentes y escuela plantean -y no al revés- y, recientemente, de vigilancia y control del gasto. En general, la participación de las familias en las escuelas comparte esta visión instrumental, suple funciones en el gasto que corresponden a los Estados y colabora en muy poco en la necesaria transformación de los sentidos actuales del aprendizaje y de la democratización de la vida escolar. Impide con ello una rearticulación del conocimiento comunitario y sus epistemes con otras desde un punto de vista dialógico y plural.

b) Formas activas y ampliadas de participación social en educación amplifican el horizonte de transformación del conocimiento y sus aprendizajes en el ámbito escolar. El caso expuesto señala cómo los aprendizajes son situados y responden a intereses que son localmente percibidos y que son complementados con estrategias que fortalecen la interlocución con el conocimiento científico y otras formas de plantear y resolver problemas, además de las locales ${ }^{8}$. En el modelo hay inserto un cuestionamiento sobre las clásicas formas de adquirir conocimiento escolar: repetición, memorización, aprendizaje dirigido y no significativo y las relaciones verticales de docentes hacia estudiantes. Muy al contrario, se fomenta el aprendizaje

8. Esta mirada, en el fondo, no parece ajena a los retos que se está planteando el sistema educativo ante las tendencias globales orientadas por competencias para la investigación, problematización y la reflexión crítica, además de la necesaria capacidad del "aprender a aprender» en un contexto cambiante y en rápida evolución. 
autónomo, autorregulado, colaborativo y autorresponsable de los y las estudiantes sin menoscabo de las identidades, prácticas culturales y lingüísticas de las familias de los niños y niñas que asisten a la escuela y sin negar otros conocimientos y realidades.

c) Apostar por un eje co-constructivo para la articulación del currículum a partir de la participación social implica posturas políticas, éticas y epistemológicas claves, pues, para ello, han de deconstruirse las jerarquías arraigadas que han determinado la interacción entre actores escolares y comunitarios en la educación pública; entre escuela como espacio de "transmisión» del saber legitimado por los cánones de la ciencia y el espacio de la vida cotidiana y de la práctica, con sus saberes; entre lenguas, la de la sociedad mayoritaria y las originarias; pero también entre las lógicas que subyacen a las diferentes expectativas hacia el futuro, donde han dominado los planteamientos asociados al progreso individual y social desde una visión más lineal, excluyente y economicista. Esto significa un distanciamiento crítico de las perspectivas del conocimiento universal que dominó la escuela moderna y que aún hoy se hacen presentes.

Sin embargo, la emergencia de otras formas de "hacer escuela» (Rockwell, 2007) y la necesaria transformación de la institución escolar en un contexto de "erosión» (Pérez Gómez, 1995) de las formas tradicionales asociadas a ella suceden en un entorno en el que perduran las estructurales y desiguales condiciones socioeconómicas que siguen afectando a los pueblos indígenas y la mayor parte de la sociedad nacional y que coadyuva al mantenimiento de ciertas tensiones entre el modelo descrito y el mayor apego a las prácticas tradicionales escolares. El modelo permite innovaciones, pero también se desarrolla entre contradicciones y reproducciones de elementos de una clásica "gramática escolar» (Tyack y Cuban, 1995).

Aun así, su esfuerzo nos permite dialogar sobre la activación, caminos, resistencias y alcances de prácticas de mayor participación comunitaria en educación y sus transformaciones sociopedagógicas. Abre horizontes para pensar, desde la experiencia, en prácticas educativas basadas en una mayor justicia cognitiva, social y cultural, pero también económica, partiendo de reestructuraciones más significativas del sistema educativo y de sus formas de comprender "lo escolar" y el papel de los diferentes actores involucrados.

El caso que hemos analizado y descrito se enmarca dentro de otras experiencias educativas mexicanas y latinoamericanas en contextos indígenas que de igual manera relacionan la participación comunitaria en educación con un debate que es tanto educativo y epistémico, como social y político. Sin poder extendernos en este artículo sobre ellas, conviene relacionar la contribución de este caso particular con estas otras experiencias en curso y que participan de dichos anhelos. Véase, entre otros, Walsh, 2013, desde la mirada ecuatoriana; Bertely, 2015 y Sartorello, 2009, para los aprendizajes generados en el trabajo con maestros indígenas de la UNEM, en Chiapas, México; González Apodaca, 2006, con los bachilleratos comunitarios en Oaxaca, México; Baronnet, 2012, para la labor de los educadores zapatistas en 
Chiapas, México; Morales, 2012, con una experiencia de educación secundaria en Cuetzalan, Puebla, México; Rojas, 2012, para la secundaria Tatuutsi Maxawkaxí en la región wixárika del estado de Jalisco, México; Jungemann y Guimarães, 2014, para la experiencia educativa del movimiento de los trabajadores rurales sin tierra en Brasil; Medina, 2015, con experiencias educativas alternativas de diversas regiones de América Latina; Molina y Tabares, 2014, para el trabajo educativo generado desde el Consejo Regional Indígena del Cauca en Colombia.

\section{REFERENCIAS BIBLIOGRÁFICAS}

BARONNET, B. (2012) Educación intercultural y descolonización del saber en la era multicultural. Revista Marista de Investigación Educativa, II (2-3), 34-42. Consultado el 17 de octubre de 2016. https://brunobaronnet.files.wordpress.com/2012/01/bruno-baronnet2012-educacion-intercultural-y-descolonizacion-del-saber.pdf.

BARRIO, J. (2005) La transformación educativa y social en las comunidades de aprendizaje. Teoría de la Educación. Revista Interuniversitaria, 17, 129-156. Consultado el 30 de noviembre de 2017. http://revistas.usal.es/index.php/1130-3743/article/ view/3118/3146.

BERTEly, M. (2000) Conociendo muestras escuelas. Un acercamiento etnográfico a la cultura escolar. México, Paidós Mexicana.

Bertely, M. (2007) Conflicto intercultural, educación y democracia activa en México. Ciudadanía y derechos indígenas en el movimiento pedagógico intercultural bilingüe en Los Altos, la Región Norte y la Selva Lacandona de Chiapas. Perú, Fondo Editorial Pontificia Universidad Católica del Perú, RIDEI, CIESAS.

Bertely, M. (2015) Enfoques postcoloniales y movimiento político y pedagógico intercultural en y desde Chiapas, México. Relaciones, 36 (141), 75-102. Consultado el 22 de noviembre de 2016. http://www.scielo.org.mx/scielo. php?script=sci_arttext\&pid=S0185-39292015000100075\&lng=es\&tlng=es.

Bolívar, A. (1999) Modelos de diseño curricular de corte crítico y postmoderno: descripción y balance, en Escudero, J. (ed.) Diseño, desarrollo e innovación del currículum. Madrid, Síntesis, 145-164.

BRISEÑO, J. (2015) Entrevistas comunitarias, la recuperación del conocimiento comunitario desde la escuela. El caso de las Secundarias Comunitarias Indígenas, Oaxaca, México. Archivos Analíticos de Políticas Educativas, 23 (95), 1-24. Consultado el 22 de marzo de 2016. http://dx.doi.org/10.14507/epaa.v23.2081.

De la Peña, G. (1995) La ciudadanía étnica y la construcción de los indios en el México contemporáneo. Revista Internacional de Filosofía Política, (6), 116-140. Consultado el 15 de octubre de 2013. http://e-spacio.uned.es:8080/fedora/get/bibliuned:filopoli1995-6-3D5E32C1AF7C-F8A8-0F8C-9B7122B9205D/ciudadania_etnica.pdf.

De la Peña, G. (2002) La educación indígena. Consideraciones Críticas. Sinéctica, (20), 46-53. Consultado el 17 de noviembre de 2016. https://sinectica.iteso.mx/index.php/ SINECTICA/article/viewFile/344/337.

DíAz BARRIgA, F. (2012) Reformas curriculares y cambio sistémico: una articulación ausente pero necesaria para la innovación. RIES, III (7), 23-40. Consultado el 7 de noviembre de 2012. http://www.redalyc.org/pdf/2991/Resumenes/Resumen_299129031002_1.pdf. 
PARTICIPACIÓN COMUNITARIA EN EDUCACIÓN-RECONFIGURACIONES DE LO ESCOLAR...

Díaz Gómez, F. (2007) Comunidad y comunalidad, en Robles, S. y Cardoso, R. (comps.) Floriberto Díaz. Comunalidad, energía viva del pensamiento. México, unam, 34-50.

Dietz, G. (2003) Multiculturalismo, interculturalidad y educación: una aproximación antropológica. Granada, Universidad de Granada, CIESAS.

Dietz, G. y Mateos, L. (2011) Interculturalidad y Educación Intercultural en México: Un análisis de los discursos nacionales e internacionales en su impacto en los modelos educativos mexicanos. México, CGEIB/SEP.

Esteva, G. (2014) La libertad de aprender. Revista Interuniversitaria de Formación del Profesorado, 28 (2), 39-50. Consultado el 25 de enero de 2016. http://www.redalyc.org/ pdf/274/27433840005.pdf.

Estrada, M. J. (2014) La prospectiva de la participación social en la educación en México: el punto de vista de los especialistas. Revista Brasileira de Educação, 19 (58), 731-753. Consultado el 25 de febrero de 2016. http://redalyc.org/articulo.oa?id=27532012011.

Favela, A. (2008) El 68 mexicano, 40 años después. Sus alcances y su vigencia. Revista Casa del tiempo, Época Iv (11-12), 91-96. Consultado el 20 de febrero de 2016. http:// www.uam.mx/difusion/casadeltiempo/11_12_iv_sep_oct_2008/casa_del_tiempo_eIV_ num11_12_91_96.pdf.

Flores, P. y Ramírez, A. (2015) La Participación Social en la Escuela en México. Una Revisión de Literatura. REICE. Revista Iberoamericana sobre Calidad, Eficacia y Cambio en Educación, 13 (3), 77-94. Consultado el 20 de febrero de 2016. http://www.uam.mx/difusion/casadeltiempo/11_12_iv_sep_oct_2008/casa_del_tiempo_eIV_num11_12_91_96. pdf.

Gigante, E. (2003) Reconocimiento y atención pedagógica de la diversidad sociocultural. Un recuento comparativo de políticas educativas, en s/A Inclusión y diversidad. Discusiones recientes sobre la educación indígena en México. Oaxaca, Fondo Editorial IEEPO, 63-91.

GONZÁLEZ APODACA, E. (2006) Etnicidad, intermediación y escuela: tres iniciativas de bachillerato intercultural en la región mixe. Tesis doctoral. México, UAm Ixtapalapa.

González ApodacA, E. y Cornelio, M. (2013) La problemática en escuelas indígenas de Oaxaca en la experiencia de sus maestras y maestros. Oaxaca, CIESAS, UNICEF.

GOnZÁlez APODACA, E. y RojAs, A. (2013) Proyectos locales, autonomía educativa y resistencia indígena, en Bertely, M.; Dietz, G. y Díaz Tepepa, G. (coords.) Multiculturalismo y Educación 2002-2011. México, COMIE, 383-414.

JimÉneZ NARANJO, Y. (2009) Cultura comunitaria y escuela intercultural. México, CGEIB/SEP. JimÉneZ Naranjo, Y. (2012) Desafíos Conceptuales del Currículum Intercultural con Perspectiva Comunitaria. Revista Mexicana de Investigación Educativa, 17 (52), 167-189. Consultado el 23 de febrero de 2016. http://www.scielo.org.mx/pdf/rmie/v17n52/ v17n52a8.pdf.

JimÉnez Naranjo, Y. y Mendoza, G. (2012) Evaluación integral, participativa y de política pública en educación indígena desarrollada en las entidades federativas. Informe de trabajo. México, DGEI.

Jiménez NARAnjo, Y. y MendozA, G. (2016) La educación indígena en México: una evaluación de política pública integral, cualitativa y participativa. Liminar. Estudios Sociales y Humanísticos, 14 (1), 60-72. Consultado el 17 de diciembre de 2016. http://www. scielo.org.mx/scielo.php?script=sci_arttext\&pid=\$1665-80272016000100005\&lng=es\&tl ng=es. 
Jungemann, B. y Guimarães, F. M. (2014) Resignificación de la educación rural desde el movimiento de los trabajadores rurales sin tierra de Brasil. Perfil de coyuntura económica, (23), 195-211. Consultado el 15 de septiembre de 2017. http://www.redalyc.org/ pdf/861/86132269011.pdf.

KreISEL, M. (2016a) De lo comunitario a lo escolar: elementos para el debate sobre la escuela comunitaria indígena. Diálogos en Educación, año 7 (13). Consultado el 29 de noviembre de 2016. www.revistascientificas.udg.mx/index.php/DSE/article/view/6454.

KreIsel, M. (2016b) Reivindicaciones del derecho a una educación propia: la figura del educador comunitario. Alteridad, 11 (1), 21-32.

KreIsel, M. (2017) Las Secundarias Comunitarias Indigenas de Oaxaca - resignificaciones de la educación escolarizada desde una propuesta alternativa para la formación de los jóvenes. Tesis doctoral. Xalapa, Universidad Veracruzana.

LEYvA, X. (2007) ¿Antropología de la ciudadanía? ... Étnica. En Construcción desde América Latina. Liminar. Estudios Sociales y Humanísticos, v (1), 35-59. Consultado el 25 de septiembre de 2013. www.redalyc.org/pdf/745/74550104.pdf.

Maldonado, B. (2000) Los indios en las aulas. Oaxaca, Centro INAH.

Maldonado, B. (2002) Autonomía y comunalidad india. Enfoques y propuestas desde Oaxaca. Oaxaca, Centro INAH, SAI, CMPIO, CEDI.

MarTínez Luna, J. (2013) Textos sobre el camino andado, tomo 1. Oaxaca, CMPIO, CAMPo, CEEESCI, CSEIIO.

Medina, P. (coord.) (2015) Pedagogías Insumisas. Movimientos político-pedagógicos y memorias colectivas de educaciones otras en América Latina. México, Universidad de Ciencias y Artes de Chiapas, Centro de Estudios Superiores de México y Centroamérica, Educación para las Ciencias de Chiapas, A.C., Juan Pablos Editor. Consultado el 15 de mayo de 2017. https://pedagogiasinsumisas.files.wordpress.com/2016/02/libro2015-patricia-medina-melgarejo.pdf.

Molina, V. A. y Tabares, J. F. (2014) Educación Propia. Resistencia al modelo de homogeneización de los pueblos indígenas de Colombia. Polis, 13 (38). Consultado el 20 de junio 2017. https://journals.openedition.org/polis/10080.

Morales, M. del C. (2012) Hacia una comunidad de práctica con enfoque intercultural: la escuela telesecundaria Tetsijtsilin en Tzinacapan, Cuetzalan, Puebla. CPU-e, Revista de Investigación Educativa, (14), 18-43. Consultado el 23 de enero de 2013. http://cpue. uv.mx/index.php/cpue/article/view/28.

OEI (1998) Documentos: VIII Conferencia Iberoamericana de Educación. Declaración de Sintra. Globalización, Sociedad del Conocimiento y Educación. Revista Iberoamericana de Educación: Monográfico: Educación, Lenguas, Culturas, (17), 251-255.

Olivé, L. et al. (2009) Pluralismo epistemológico. La Paz, Clacso, Cides-umsa, Muela del Diablo, Comuna.

Paraskeva, J. (2001) El Currículum como Práctica de Significaciones. Kikiriki, Cooperación Educativa, (62-63), 8-16. Consultado el 15 de mayo de 2012. http://www.quadernsdigitals.net/datos_web/hemeroteca/r_7/nr_498/a_6791/6791.html.

Pedro, F. y Rolo, F. (1998) Los Sistemas Educativos iberoamericanos en el contexto de la globalización. Interrogantes y oportunidades. Revista Iberoamericana de Educación: Monográfico: Educación, Lenguas, Culturas, (17), 257-289.

Perales, F. y Escobedo, M. (2016) La participación social en la educación: entre propuestas innovadoras y tradición educativa. REDIE, 18 (1), 69-81. Consultado el 10 de agosto de 2016. http://www.investigacionenlaescuela.es/articulos/26/R26_1.pdf. 
PÉrez Gómez, A. (1995) La escuela, encrucijada de culturas. Investigación en la Escuela, (26), 7-24. Consultado el 10 de agosto de 2016. http://www.investigacionenlaescuela. es/articulos/26/R26_1.pdf.

Rivera, L. (2016) Autonomía de gestión de las escuelas: la ilusión del poder de decisión. Nexos. Consultado el 12 de noviembre de 2016. http://educacion.nexos.com. $\mathrm{mx} / \mathrm{p}=125$.

ROCKWELL, E. (2005) La apropiación, un proceso entre muchos que ocurren en ámbitos escolares. Memoria, Conocimiento y Utopía, Anuario de la Sociedad Mexicana de Historia de la Educación, 1 (1), 18-38.

ROCKWELl, E. (2007) Hacer escuela, hacer estado. La educación posrevolucionaria vista desde Tlaxcala. Zamora, Michoacán, El Colegio de Michoacán, CIESAS, CINVESTAV.

ROCKWELl, E. y EZPELETA, J. (1983) La escuela: relato de un proceso de construcción teórica. Ponencia Seminario Clacso. São Paulo, Brasil.

ROJAs, A. (2012) Escolaridad y política en interculturalidad: los jóvenes wixaritari en una secundaria de huicholes. Guadalajara, Editorial Universitaria UdeG.

Ruiz, A. y Quiroz, E. (2014) Educación comunitaria: una propuesta alternativa para los pueblos indígenas de Oaxaca-México. Polis, 13 (38). Consultado el 30 de octubre de 2014. http://polis.revues.org/10107.

SARTORELLO, S. (2009) Una perspectiva crítica sobre interculturalidad y educación intercultural bilingüe: El caso de la Unión de Maestros de la Nueva Educación para México (UNEM) y educadores independientes en Chiapas. Revista Latinoamericana de Educación Inclusiva, 3 (2), 77-90. Consultado el 13 de marzo de 2016. www.rinace.net/rlei/ numeros/vol3-num2/art5.html.

SchmeLKes, S. (2003) Educación intercultural. Reflexiones a la luz de experiencias recientes. Sinéctica, (23), 26-34. Consultado el 3 de diciembre de 2016. http://www.redalyc.org/ pdf/998/99815908005.pdf.

SiLva, T. (1999) Documentos de Identidad. Una introducción a las teorías del currículo. Belo Horizonte, Autêntica Editorial.

TORRES, J. (2010) La justicia curricular. El caballo de Troya de la cultura escolar. Madrid, Morata.

TREVIÑo, E. (2015) La Educación Superiory el advenimiento de la sociedad del conocimiento. Ciudad de México, Asociación Nacional de Universidades e Instituciones de Educación Superior.

Tyack, D. y Cuban, L. (1995) Tinkering Towards Utopia: A Century of Public School Reform. Cambridge, Harvard University Press.

Velasco, H. y DíAz de RADA, A. (1997) La lógica de la investigación etnográfica. Un modelo de trabajo para etnógrafos de escuela. Madrid, Editorial Trotta.

WALSH, C. (2012) Interculturalidad y (de)colonialidad: Perspectivas críticas y políticas. Revista Visão Global, 15 (1-2), 61-74. Consultado el 6 de septiembre de 2013. http:// editora.unoesc.edu.br/index.php/visaoglobal/article/viewFile/3412/1511.

Walsh, C. (ed.) (2013) Pedagogías decoloniales. Prácticas insurgentes de resistir, (re)existir $y$ (re)vivir, tomo 1. Quito, Abya-Yala.

Wolcott, H. (1993) Sobre la intención etnográfica, en Velasco, H.; García Castaño, F. y DíAz DE RADA, A. (eds.) Lecturas de antropología para educadores. Madrid, Editorial Trotta, 127-144. 
Zurita, U. (2011) Los desafíos del derecho a la educación en México a propósito de la participación social y la violencia escolar. Revista Mexicana de Investigación Educativa, 16 (48), 131-158. Consultado el 14 de febrero de 2016. http://www.scielo.org.mx/scielo. php?script=sci_arttext\&pid=S1405-66662011000100007\&lng=es\&tlng=es. 